\title{
Rule-based Recommendation System based on Semantic Web of Things
}

\author{
Ahmed Salama ${ }^{1}$, Masoud E. Shaheen ${ }^{2}$, Haytham Alfeel ${ }^{3}$ \\ ${ }_{1,2,3}$ Faculty of Computers and Information, Fayoum University, Egypt. \\ 1,2,3 ORCIDs: 0000-0002-1781-4217, 0000-0003-4853-3415, 0000-0002-8416-0292
}

\begin{abstract}
Social networking has certainly been the most apparent concept of virtual human life where all users may communicate with each other. Thus all users of social networks aim to handle and monitor the demands of their lives through their platforms for virtual lifestyle. And since the Internet of Things is now considered one of the latest technologies which connect everything around us together as objects in the form of an IP address that enables contact between them. On the other side, IoT also struggles from the variability of data obtained from various sensors. Addressing this problem includes the alignment of semantic web technology. We, therefore, aim to take advantage of the Internet of Things and Semantic Web technologies revolution by developing an integrated model based on the semantic web. Through our proposed research, we intend to create a rule-based recommendation framework that advises users to take the most convenient action that IoT applications should execute on their current social network activities utilizing the built-in definition logic rules. By virtue of this social recommender, Users of IoT applications can monitor their apps only by communicating their social status via their social media instead of using special IoT apps on their mobile devices. As a result, there is more integration between IoT resources and Social Network resources.
\end{abstract}

Keywords: Semantic Web, Internet of Things, Semantic Modelling, Rule-based Recommender, Semantic Resigning, Description Logics, Sensors

\section{INTRODUCTION}

Social networking has been used as the key portal that people around the world choose to communicate and exchange knowledge in various forms. Where efforts have been made to improve the usability of these social platforms. Else, Social users nowadays manage their business and different aspects of their life through social media. But till now there aren't fully functional social platforms available that facilitate the controlling of surrounded devices smoothly just via their social accounts. Just by integrating the IoT with social media techniques, which can lead to increasing the scalability of social networks. This because of the ability of IoT [1] to control the gathered information of different devices over the Internet. But, unfortunately, fetched information gathered from the sensors and actuators suffers from lacking interoperability and heterogeneity problems. According to the different types of data and sensors used in IoT applications. Semantic Web technique [2] can be used to organize and model the different heterogeneity data gathered from sensors and actuators in a structured format to facilitate the processing tasks on these fetched data. Hence, through our research, we propose firstly a new Semantic Web ontology-based that used to model and organize data fetched from the IoT sensors and actuators to enrich the meaning of this data. Our new proposed ontology is extended from the standard Semantic Sensor Network (SSN) ontology [3] that was declared to solve the main issue of heterogeneity. After handling the main concern of the IoT technology using semantic modelling techniques. We integrate this modelling IoT data with the Social Networking platforms to build a new smart social network recommender. The proposed recommender could recommend the suitable action to be performed by IoT actuators that control the Applications owned by the social media users. Hence, according to their concurrent status that appears in the form of posts or tweets simultaneously. Depending on the proposed recommender social media users could express their statuses on their social accounts. Hence, our smart recommender will recommend the best-performed action by the actuators of their IoT applications. We use the sentiment analysis technologies [4] and our proposed reasoning tool aiming to build a robust intelligent social media platform aided with the features of Internet-of-Things. The rest of this research is structured as the following: The technologies which we use through our work are illustrated in Section II. Also, section III presents the Literature Review works that fit our proposed works. Our proposed architecture is discussed through Section V. Section VI presents our implementation and processes. Also, Section 6 presents our results. While the evolution of our proposed architecture is illustrated in section VII. Last but not the least, section VIII concludes our paper and discusses our objectives for future work. 


\section{RELATED WORKS}

In this section, we show a set of different related researches thorough which authors investigate the relation between Internet-of-Things and Semantic Web techniques. Hence we took in our consideration these efforts to do better through our works. In the beginning, Sensor Web Enablement (SWE) group [5] has developed a set of standards to describe sensors and their data such as SensorML3. The SensorML3 is a language to describe sensors, Observations, and Measurements (O\&M) [6]. However this language provides important syntactic descriptions using XML, it suffers from lacking the expressability, interpretation, and interoperability provided by ontology languages such as OWL. Also, The Semantic Sensor Networking (SSN) Ontology describes the sensors and actuators' data, such as outputs, observation values. While it delivers a more detailed description of subjects, properties, and instances that provides full descriptions for a wide range of applications, but it still suffers from the latency of query processing due to having different components that maybe not useful to be useful every time. Additionally, a huge amount of sensor metadata and measurements is published based on a set of different measurements and observations which mapped to the semantic format such as the Resource Description Framework (RDF) [7]. On the other hand, the contribution of [8] aimed to structure the establishment of an IoT architecture based on social platforms of human beings. Where the Pachube platform enables web developers to access sensor data to build applications. In addition to that, the effect of semantic web technologies on social media explained clearly [9] as a way to increase the understanding of social behavior and its metrics, such as its effect which influence engagement between social media users, and enrich the knowledge of effect by applying the semantic web technologies. Also, authors in [10] proposed a semantic social behavior extraction process based on the most commonly used social networks, such as Facebook that enable the extraction of semantic behaviors from any semantic information. Besides, learning environmental systems based on social network platforms is introduced in [11] to perform an automotive semantic reasoner that depends on the interactions among people and their relations with the learning resources. They mixed the analysis of social networks using the semantic concepts and the analysis of social graphs by integrating the processing of semantic knowledge. Also, the Recommender System in [12] employs textual data from different social network platforms such as Twitter or Facebook to recommend specific items for new users. By collecting users' posts, comparing them with their relevant products, and generate recommendations according to the collected data. Also, Authors in [1] developed a new conceptual method for the exploitation of the services for the Social IoT among different IoT applications. They conclude a set of social relationships between people-and-things and between things-and-things to deliver a group of different recommendation services. Besides, Authors in [2] proposed an integration model of sensor networks with social networks. This proposed model enables applications to sense the behaviour of a social user in much better ways and then provides more personalized solutions. They depended on their proposed applications into making their deeper integration with the user's daily life through social platforms and sensor network data. Last but not least, authors in [3] proposed a set of appropriate rules and policies to build the management and integration of the social networks among different objects to enhance the navigability of the social network. Also, they defined a new IoT architecture that includes the functionalities needed to integrate IoT objects with the social network.

\section{BACKGROUND TECHNIQUES}

We present through is section set of various technologies that we used during our development to achieve our proposed works such as IoT, Semantic Web Reasoning, sentiment analysis techniques, and the recommendation system techniques.

\section{III.I Internet of Things}

IoT Gateway plays a vital role in smart applications to facilitate the flexible integration of wireless sensors, mobile communication networks management, and the control of wireless sensor networks. The IoT architecture is divided into four phases, where the first phase represents the sensors and actuators' devices for connecting everything and hence providing users with actual data that represents the main purpose of the Internet of Things. On the other hand, the second phase represents the data acquisition process that fetches the data of sensors and actuators used in smart applications through the IoT gateways that preprocessed the gathered data locally before sending it to the cloud layer also, it provides set of security features for the IoT network and its transmitted data by managing and controlling the information moving in both directions, Hence it can protect data transmission to the cloud from leaks and IoT devices from being attacked by malicious outside attacks. Too, the third phase or the edge analytics layer that provides the applications with faster response times and more flexible processing and analysis of IoT data. Finally, the fourth phase of IoT architecture represents the cloud data storage model that is designed for storing and processing massive volumes of data fetched from different sensors and actuators [13] as shown in Figure.1.

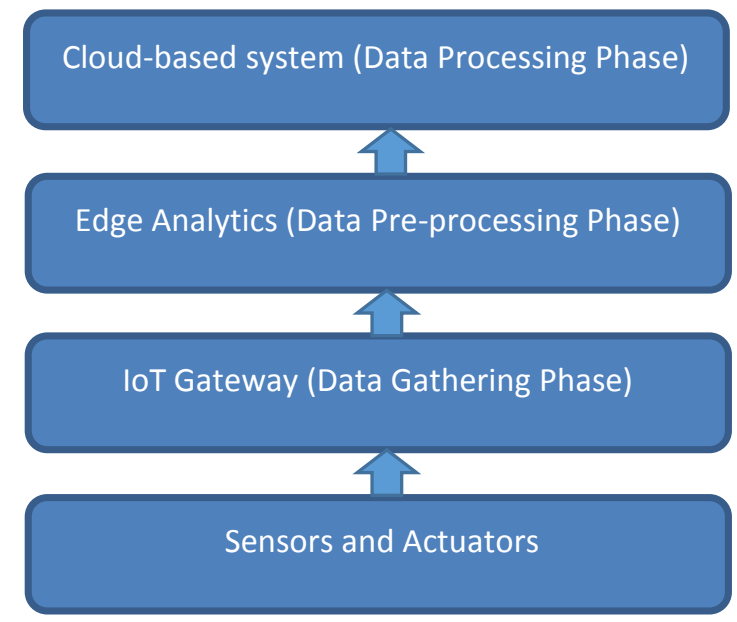

Fig. 1. Internet of Things Architecture 
International Journal of Engineering Research and Technology. ISSN 0974-3154, Volume 13, Number 6 (2020), pp. 1455-1465

(C) International Research Publication House. https://dx.doi.org/10.37624/IJERT/13.6.2020.1455-1465

\section{III.II Semantic Reasoning}

Semantic Web reasoning is the process of inferring the set of new relations from a collection of given propositions condition. It is related to the OWL Description Logic (DL) used as a Knowledge Representation Languages. KR depends on artificial intelligence tools and semantic web concepts. By using the reasoning techniques, we could derive new facts that are not explicitly expressed or inferred in ontology or in knowledge base. It dedicates a piece of new information based on the given and relevant concepts of terminological knowledge of applications. In this context, Semantic reasoner tools used to model sensors data within an ontology environment to infer new information to enrich the given pure and data. These reasoning techniques are formed into the Rulebased layer which is defined on the top of the new Semantic Web architecture, on which different rule languages defined for building different expert systems such as SWRL (Semantic Web Rule Language) as shown in Figure.2.

\section{III.V Sentiment Analysis Techniques for Social Media}

Social media, such as Twitter and Facebook, have a significant art in people's lives. They reflect the contact of users with each other via the sharing of their personal or public knowledge and opinions. It is also listed as the fourth most common resource for accessing concurrent knowledge. Different experiments have been performed on social network data to clarify their user's behaviour and to establish various comprehensive management plans [16]. In addition, Sentiment analysis is one of the most relevant strategies utilized today in various disciplines in our lives [17] relying on supervised machine learning strategies cantered on dataset evaluation. Our research aims to empower social networking functionalities and integrate it with the Internet-of-Things technology to analyse tweets simultaneously to categorize sentiment of specific users. This will help us to build a robust and smarter recommender system based on the sentiments of social media users to recommend actions for actuators of IoT applications that are located in the same locations where users post their tweets.

\section{III.VI Recommendation System techniques}

Recommender systems play an important role in delivering information and services to users depending on their interest based on data mining techniques. Also, Recommender systems are used to handle information overloading issues by studying the users' preferences. Recommender System techniques have been classified into various categories such as content-based filtering, collaborative filtering, knowledge, and location-based techniques. However, Recommender Systems still suffer from different issues such as dynamism, data variety, trust, and privacy which we tried to handle such of them based on our training data set that gathered from the sensors data and social' user behaviours or posts. It is reported that the recommendation system is considered one of the most needed research topics that combine different science together such as Artificial Intellegence, data mining, and information retrieval especially when we try to integrate it with recent technologies such as Semantic Web and Internet of Things. On the other hand, the recommendation system architecture is divided into three main phases; the data collection phase, the recommender engine processing phase, and finally the recommendation system's user gateway as shown in Figure 3.

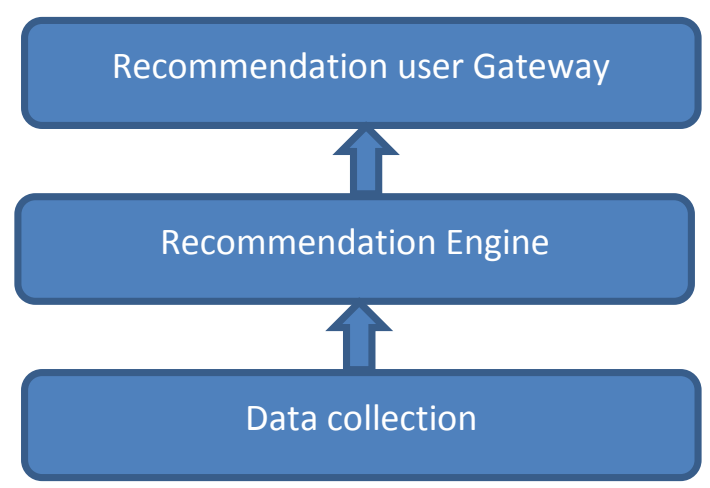

Fig. 3. Recommendation System Architecture

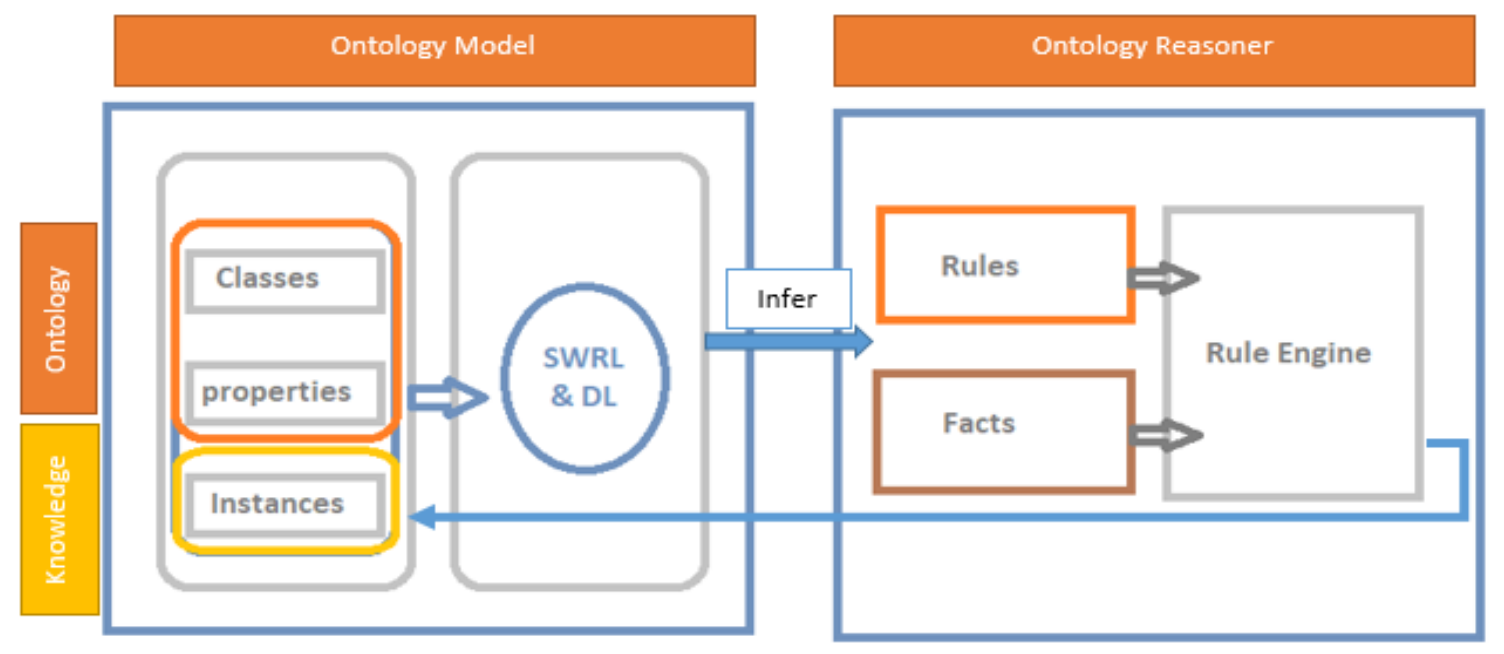

Fig. 2. Semantic Web Reasoning Architecture 
International Journal of Engineering Research and Technology. ISSN 0974-3154, Volume 13, Number 6 (2020), pp. 1455-1465

(C) International Research Publication House. https://dx.doi.org/10.37624/IJERT/13.6.2020.1455-1465

\section{PROPOSED WORK DISCUSSION}

The main contribution behind this work is to build a new automotive and smarter recommender system to improve the usability and flexibility of the IoT resources in human life. This could be delivered by integrating the social network technologies IoT resources and techniques via the semantic web technologies. Through our proposed recommendation architecture, we could track the synchronous social behavior of social network users such as posts or tweets, and then our smarter recommender can recommend a new action to his IoT application to perform at the same time. This smarter recommender will depend on the shared status from users and then control their own IoT applications automatically. The proposed architecture is divided into four layers as shown in Figure.4.

Where we collect the data of different types of IoT sensors in real-time such as lighting, temperature, and air conditioner through the WI-FI protocols of IoT such as Arduino Node MCU as a pre-processing layer of our architecture through the First Layer.

While, we applying a sentiment analysis algorithm to perform an accurate analysis of the fetched tweets and categorized them into the proper ontology classes simultaneously as a pre-processing phase.

The Third Layer represents the layer of processing the modelled IoT ontology by extracting more knowledge and information from the gathered data of sensors. This could be performed by using the reasoning language such as Description Logics (DL) to infer new relations and rules that could enrich the value of our proposed model of IoT architecture.

The top layer represents the recommendation phase where the inferred semantic relations based on the current sensor status and the real-time streaming tweets from users who are in the same locations of fetched sensor's data. This layer provides an automotive rule-based recommender that depends on the social filtering techniques and sentiment analysis algorithm.

This recommender could recommend automatically the proper actions for actuators of the IoT applications based on the givens of sensors and social data as well as the proposed reasoning semantic Rules.

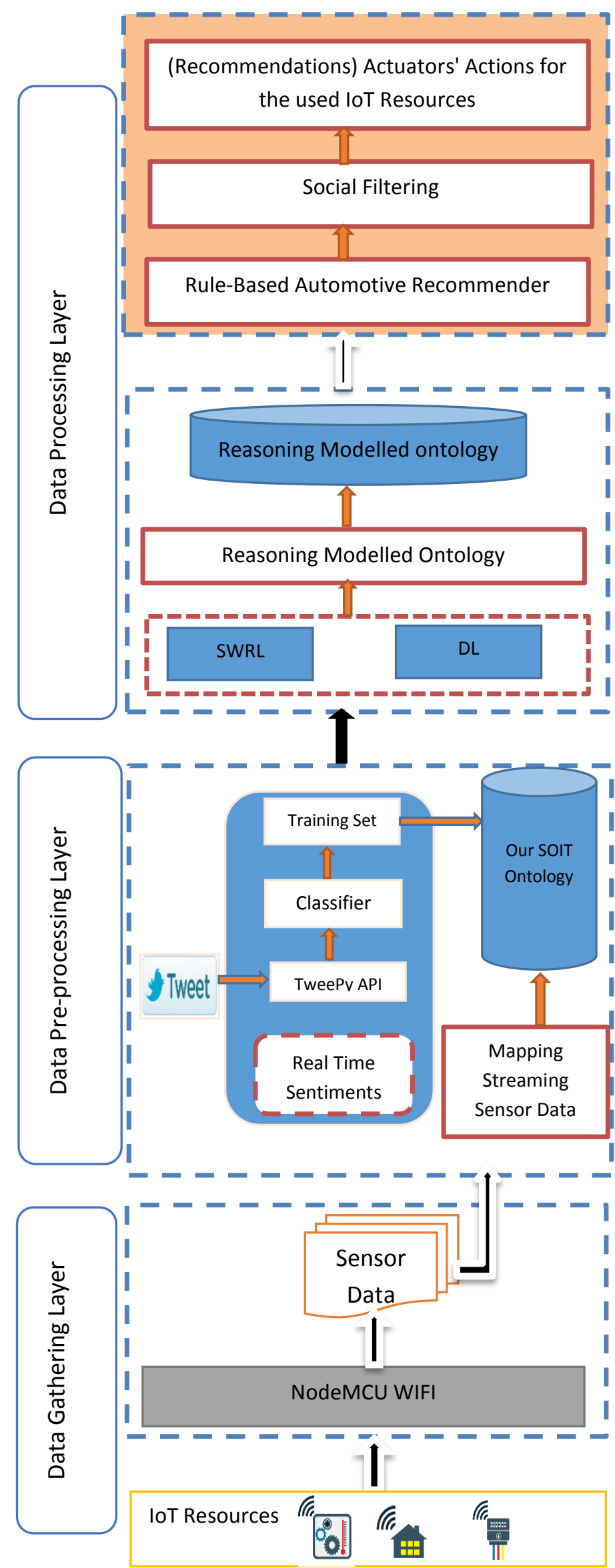

Fig. 4. Our Proposed Recommender System Architecture 


\section{IMPLEMENTATION}

In this section, our proposed algorithm is implemented via different technologies such as Sentiment analysis python techniques, firebase as a mobile database management system, Semantic Protégé as an ontology IDE and knowledge-based environment which could be used as the main tool for building OWL ontologies, Semantic Web Rule Language (SWRL) and sentiment analysis algorithm. In addition to that the IoT Resources such as NodeMCU wireless module for configuring the IoT sensors, actuators like Light Dependent Resistor (LDR) sensor for detecting the light mode, $\operatorname{lm} 35$ temperature sensor, GPS location sensor, and IR Transmitter sensor.

All of these are developed across the Arduino toolkit with the integration of python programming language. The proposed algorithm fetched different sensors data resources and then stored them into real-time database storage such as Firebase to update them simultaneously. After that, the stored sensor data are mapped from the Firebase Relational Database into our new proposed ontology using mapping algorithms such as R2RML [19] as a Mapping Language.

On the other hand, our algorithm tracks the current status of users who are affected by the modelled sensors through our ontology. This could be done by conducting sentiment analysis and mining algorithms to detect it at a time and then inserting it also into our new ontology which is modelled to link the sensors' resources data with its affected users. Hence, our proposed ontology could store the streaming tweets gathered from the social media and the sensors' values of the IoT applications used by the same user who shared the concurrent social media tweet to be processed using semantic web technologies such as the Description Logics. The DL is one of semantic web reasoner languages that are used for building a reasoning ontology provided with new inference relations based on given semantics' logic by building a knowledge base model. It could reason the sensors data through our ontology based on the terminological box (TBox) and assertion box (ABox) where the TBox describes the main concepts and relations between them whereas the ABox describes the actual values of individuals or the fetched data from the sensors [20] as shown in Figure .5.

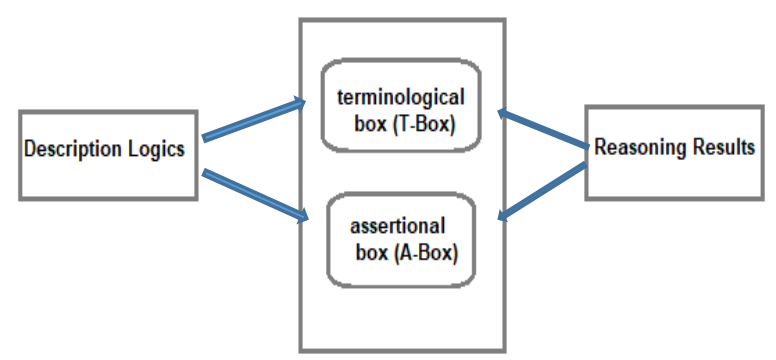

Fig. 5. Description Logic and Reasoning Architecture
Also, This Description logic model of our ontology is integrated with Semantic Web Rule Language (SWRL) to generate new inference rules based on the captured data form both sensors' resources and social media users. The SWRL rules are used to improve the expressiveness of the ontology models by reasoning on the instances of ontology concepts and classes in our social IoT ontology and infer new knowledge and relations between our modelled ontology classes. It depends on the integration between OWL DL and the Unary and Binary of the Rule-based Mark-up Language. The rules are also formatted in an antecedent-consequent fashion initiated from zero or more atoms. Based on the former ontologies, In addition to that, SWRL rules had been created to map different individuals of one ontology into the other and then infer a set of new relationships between similar classes with different descriptions.

Hence, these new inferred relations used to build our new smart auto recommender system according to the social behaviour of users and the current sensor data of their surrounded environment based on the common location for both sensors and users to ensure that the status of users which shared through social networks are matched with the current sensors data that are detected and stored into an ontology simultaneously. Users share their feelings about the weather if they are hot for example through his twitter account, so the LM35 temperature sensor detects that the temperature degree is highly recommended automatically to reduce the degree of the air conditioner of his room through the used actuators through our proposed semantic ontology as inferencing rule as shown in Figure 6.

After applying the proposed algorithm, our developed ontology could dynamically read and write the streaming data from both sensors and users who share their status through twitter and also use the IoT applications which are affected by the sensor readings. This will enable us to build our proposed rule-based recommender system which could help users in controlling their IoT applications by using their social platforms.

\section{RESULTS}

Through this section, our implementation results of the proposed algorithm and architecture are illustrated. One of the most important results is unifying and modeling different sensors' objects in one ontology as shown in Figure.7 stored simultaneously in Semantic Web format. The ontology has a prefix value named SIOT and it is divided into a set of classes that define the Sensors values and types such as temperature, lighting, and air conditioner sensors. 
International Journal of Engineering Research and Technology. ISSN 0974-3154, Volume 13, Number 6 (2020), pp. 1455-1465

(C) International Research Publication House. https://dx.doi.org/10.37624/IJERT/13.6.2020.1455-1465

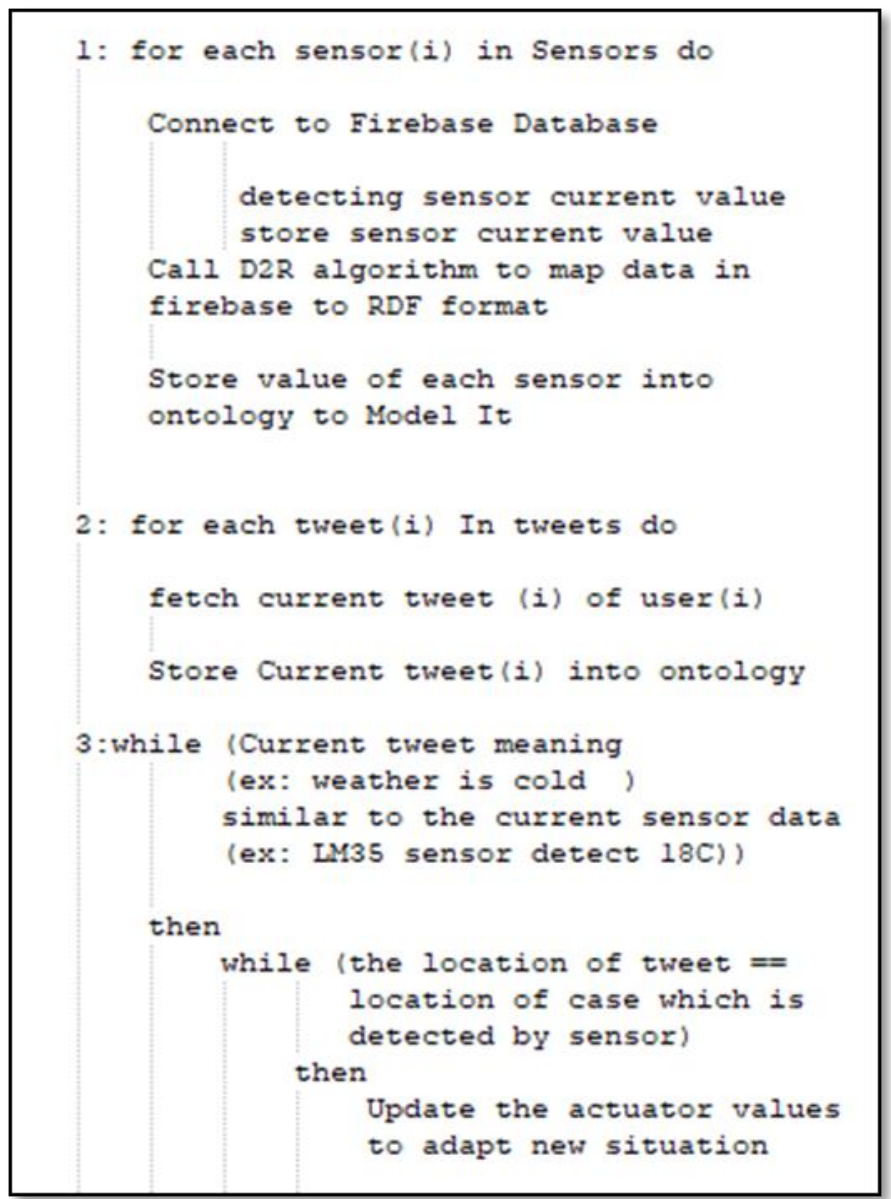

Fig. 6. Proposed Recommender System Algorithm into RDF format and inserted into the ontology that makes it streaming sensor ontology as shown in Figure.8.

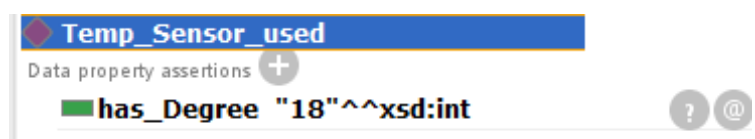

Fig. 8. Mapped Sensor Values into Ontology

Also, our smart recommender system has been developed based on Description Logics (DL) that depends on the First Order Logic (FOL) and integrates with the Semantic Web Rule Language (SWRL) that depends on the Web Ontology Language (OWL). This integration leads to new inference rules that use the IoT sensor data and social tweets to build the new smart recommender system. Where the DL rules and SWRL rules reflect that for each user and for each temperature degree allocated from the sensors used in the IoT applications that are used by this specific user. In case this user posts a tweet referring to his feeling about the weather. the proposed logic for this case will compare this tweet with the actual temperature degree that is retrieved from the sensor that is located in the same place of the tweet, So if the comparison returns true then our recommender system sends automatically a suitable action for the actuators of the IoT application to be executed as shown in Figure 9.and Figure.10.

It includes also a set of observation values and Person class which includes values of users who use the IoT's applications and shared their statues at the same time. This by fetching them into the Firebase database management system in JSON structured formant. And then these stored values are mapped

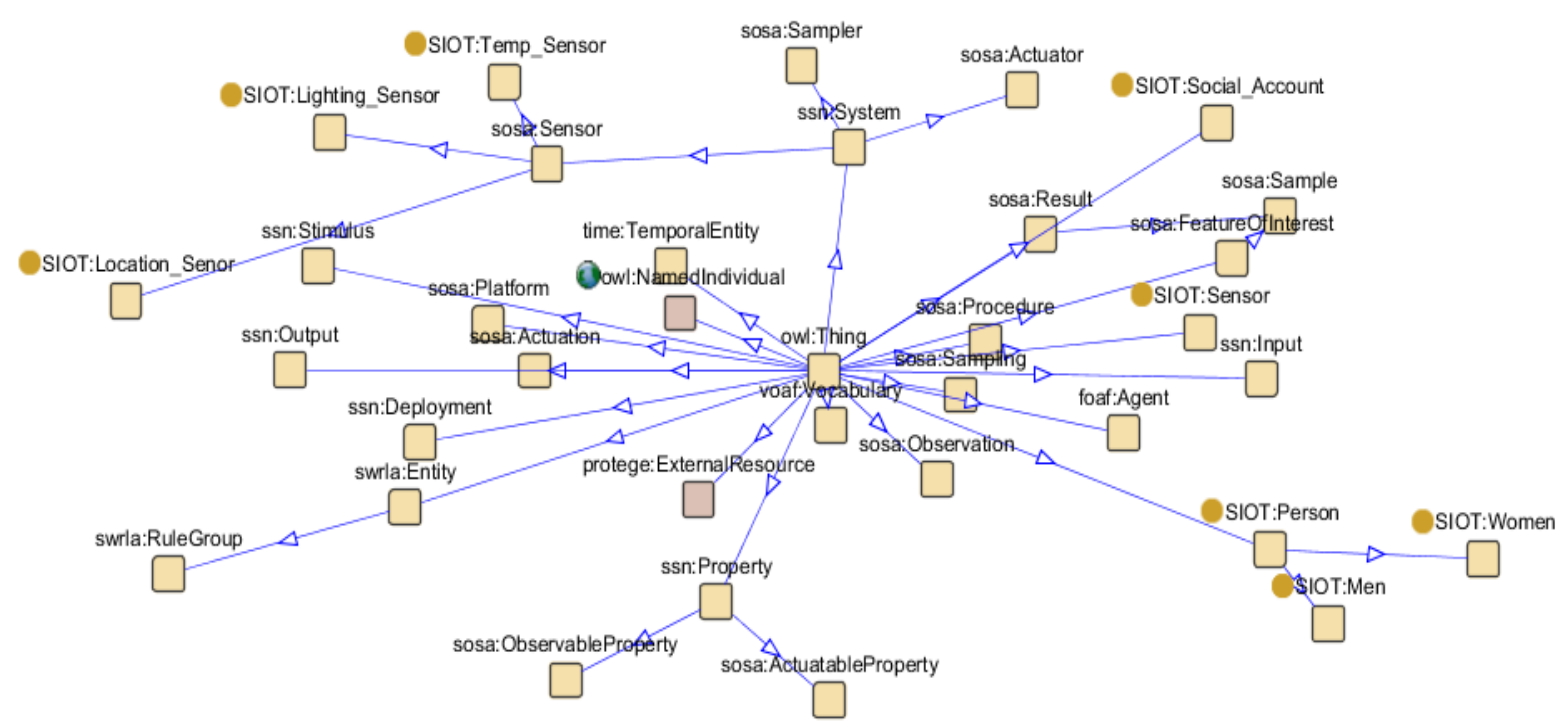

Fig. 7. Proposed SIOT Ontology 
International Journal of Engineering Research and Technology. ISSN 0974-3154, Volume 13, Number 6 (2020), pp. 1455-1465

(C) International Research Publication House. https://dx.doi.org/10.37624/IJERT/13.6.2020.1455-1465

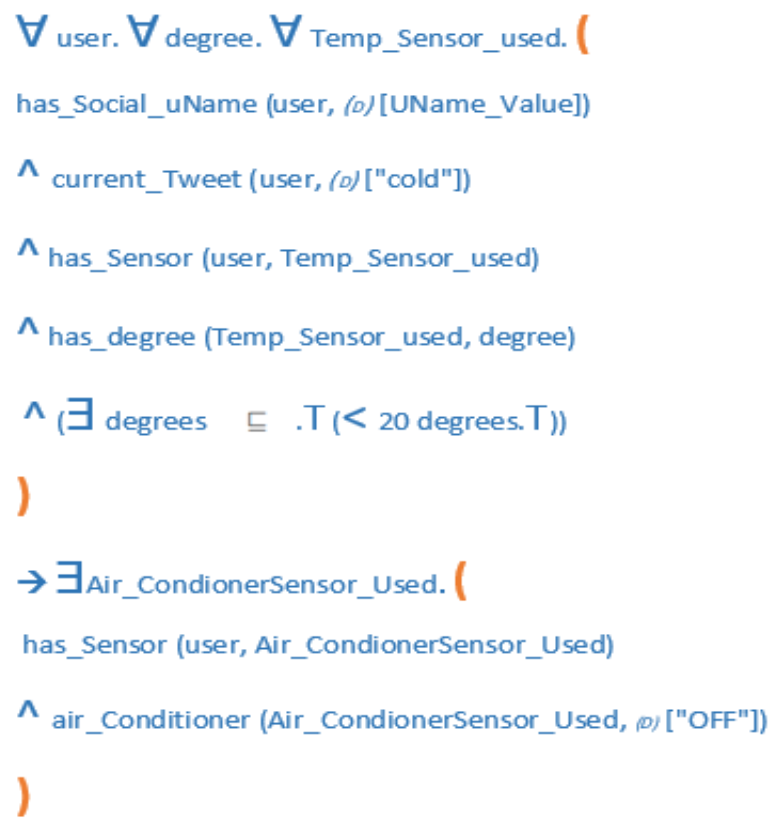

Fig. 9. DL Rules for our Smarter Recommender system

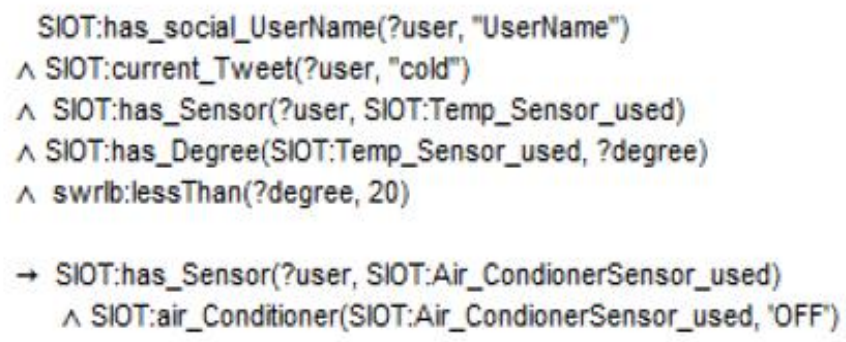

Fig. 10. SWRL Rules of Proposed Recommender

The ontology has a prefix value named SIOT as an abbreviation for the Social Internet of Things ontology and it is divided into a set of classes that define the Sensors values and types such as temperature, lighting, and air conditioner sensors. It includes also a set of observation values and Person class which includes values of users who use the IoT's applications and shared their statues at the same time. This by fetching them into the Firebase database management system in JSON structured format as shown in Figure.11. And then these stored values are mapped into RDF format and inserted into the ontology that makes it streaming sensor ontology as shown in Figure.12 and Figure.13

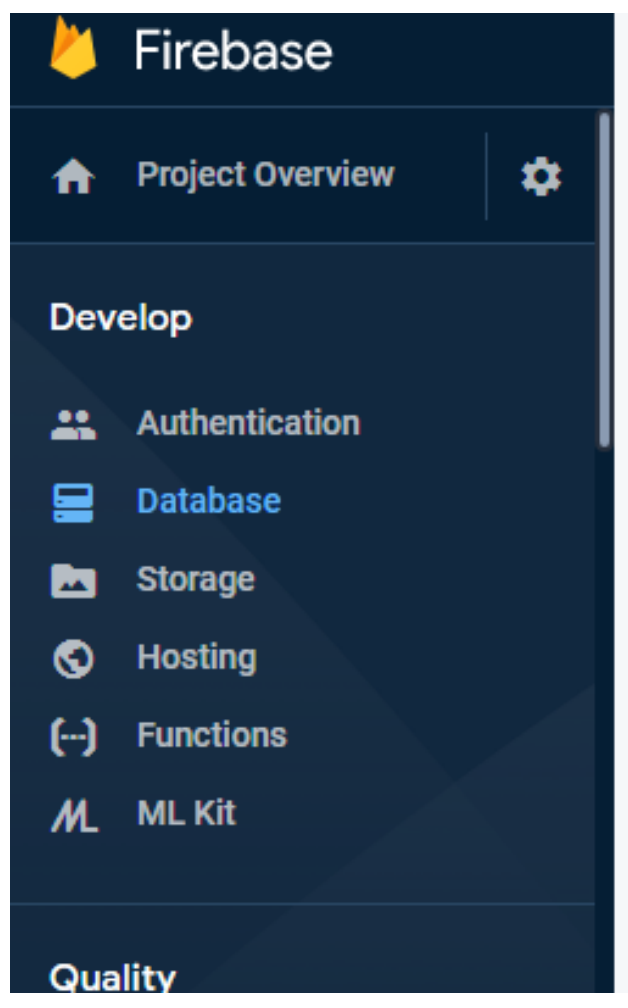

\section{Database 븜 Realtime Database}

\section{Data Rules Backups Usage}

\section{G) https://iotphd.firebaseio.com/}

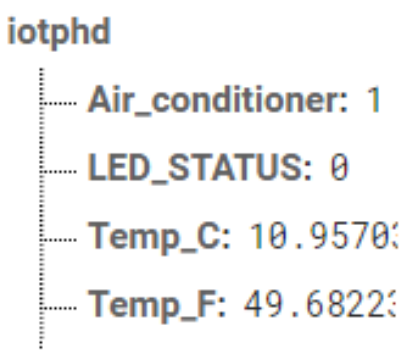

Fig. 6. Streaming IoT sensors' values for a smart home applications in Firebase Real-time Database 


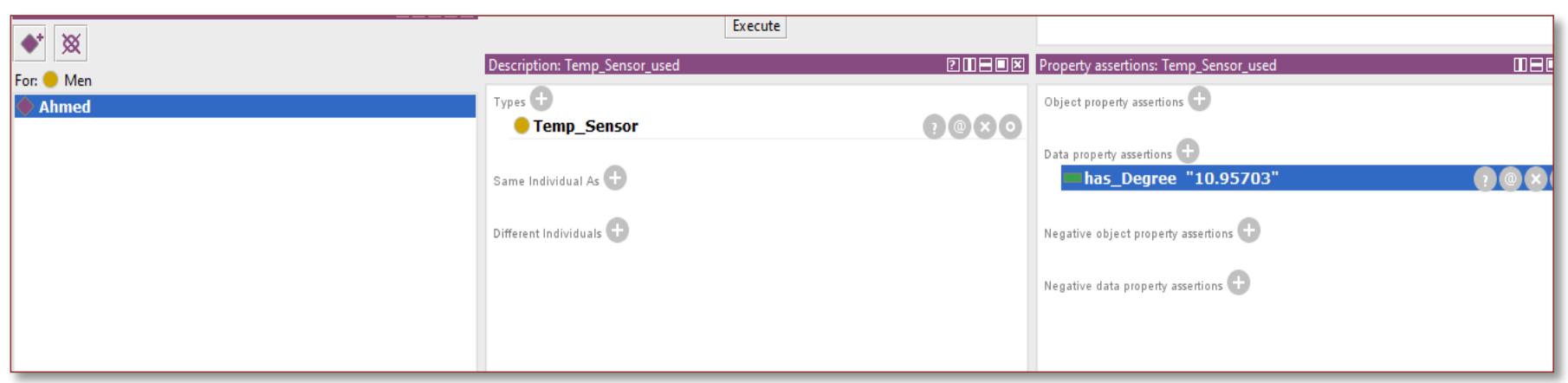

Fig. 12. Updating our ontology model with the streaming Temperature Degree

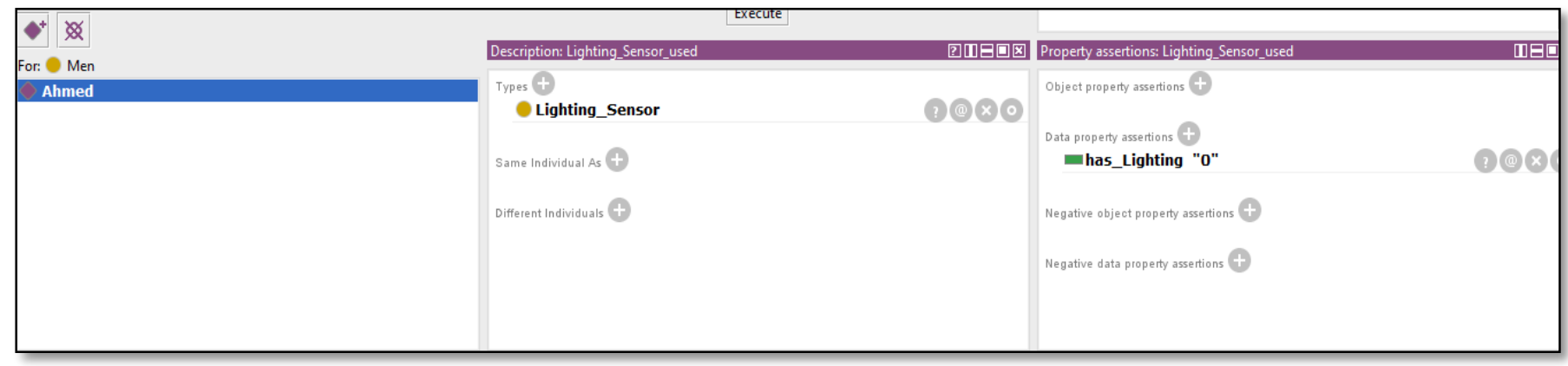

Fig. 13. Updating our ontology model with new value for the lighting using reasoning rules and send it to actuators to be stored into firebase

On the other hand, after applying the Sentiment analysis algorithm of our architecture to track the most recent tweet from a specific user who is in the same location where the IoT application sends its data to ontology. The fetching tweet is stored into an ontology and updated every time the user changes his status on Twitter as shown in Figure 14 and Figure 15. The gained information extracted from user tweets by sentiment analysis is then transmitted to corresponding sensors.

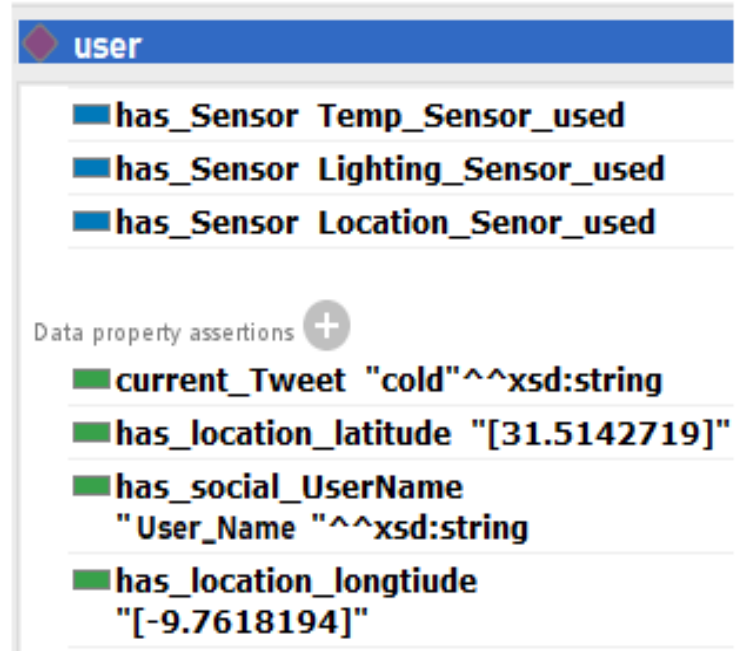

Fig. 7. Imported Social media user data into ontology

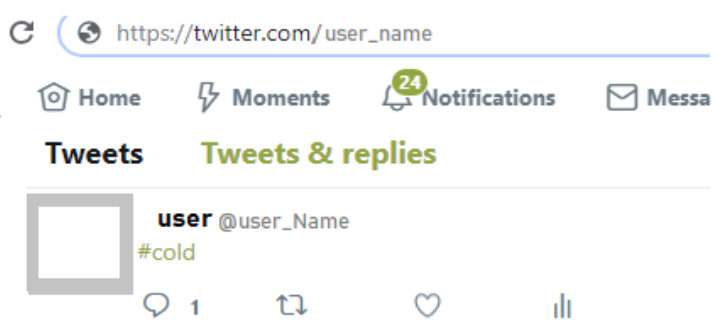

Fig. 8. Streaming Tweet for Specific User

This smart recommender that based on the proposed reasoning model conducts the recommended actions to be performed through actuators based on the current social status the twitter's users shared making their surrounded environment suitable and more flexible just by turning his physical environment based on what expressions and feelings he texted it through social media as shown in Figure 16.

\section{Air_conditioner_Senser_used}

\section{Data property assertions \\ Air_Conditioner "OFF"}

Fig. 9. Value of Actuator which is recommended by proposed recommender according to the current status of the user and the value of temperature sensor (LM35) 
International Journal of Engineering Research and Technology. ISSN 0974-3154, Volume 13, Number 6 (2020), pp. 1455-1465

(C) International Research Publication House. https://dx.doi.org/10.37624/IJERT/13.6.2020.1455-1465

\section{EVALUATION}

Through this section, set of evaluation methods were performed by conducting an practical experiment to measure and evaluate our proposed recommender systems based on the accuracy metrics such as precision-recall and F-Score that reflects to which extent, the accuracy of our recommendations fit the real users' feeling that is expressed by the shared status of these users who use social media based on the confusion matrix [21] as shown in Table 1.

Table 1. Confusion Matrix Diagram

\begin{tabular}{|l|c|c|}
\hline & Positive & Negative \\
\hline Positive & TP & FP \\
\hline Negative & TN & FN \\
\hline
\end{tabular}

The Precision reflects the value of the relevant instances from all the retrieved instances, whereas recall reflects the value of all relevant instances. On the other hands, the F1 score is the value of combining recall and precision using the harmonic mean. Hence, we could measure the sensitivity, accuracy and relevance factors based on the measurements of both Precision and Recall as shown in the following Equations.

precision $=\mathrm{TP} /(\mathrm{TP}+\mathrm{FP})$

Recall $=\mathrm{TP} /(\mathrm{TP}+\mathrm{FN})$

F-score $=2 *($ Precision $*$ Recall $) /($ Precision + Recall $)$

Accuracy $=(\mathrm{TP}+\mathrm{TN}) /(\mathrm{TP}+\mathrm{TN}+\mathrm{FP}+\mathrm{FN})$

Through this part, we examined the accuracy of our proposed rule-based recommender system by evaluating the precision, recall and the F-score of the recommender system to get accurate insights which could reflect what extent our proposed recommendation system is accurate. This experiment has been done on the status of the actuators which control the air conditioner or Light system. Where the results will be either ON or OFF mode.

So we used the binary confusion matrix to calculate these proposed accuracy factors. It is reported that we have a training set which includes around 10 of the expected values among ON or OFF based on the semantic reasoning rules as well as test set based on the predicted results which are returned according to the usage of our recommender system as shown in Table 2 and Table 3..

Table 2. Confusion Matrix Results

\begin{tabular}{|l|l|l|}
\hline Confusion Matrix & ON & OFF \\
\hline ON & $\mathbf{4}$ as $\mathbf{T P}$ & $\mathbf{1}$ as $\mathbf{F P}$ \\
\hline OFF & $\mathbf{1}$ as $\mathbf{F N}$ & $\mathbf{4}$ as TN \\
\hline
\end{tabular}

Table 3. Our Smarter Recommender Evaluation Measurements

\begin{tabular}{|c|c|}
\hline Expected & Predicted \\
\hline $\begin{array}{c}\text { Tweet is Hot \&\& Temp }>25 \\
\& \& \text { user and sensors are in } \\
\text { the same Location }-> \\
\text { (ON) }\end{array}$ & $\begin{array}{c}\text { Tweet is Hot_Choc \&\& } \\
\text { Temp }>25 \& \& \text { user and } \\
\text { sensors are in the same } \\
\text { Location } \rightarrow \\
\text { (OFF) }\end{array}$ \\
\hline $\begin{array}{c}\text { Tweet is Hot } \& \& \text { Temp }>25 \\
\& \& \text { user and sensors are in } \\
\text { the same Location } \\
->\quad(O N)\end{array}$ & $\begin{array}{c}\text { Tweet is Hot } \& \& \text { Temp }>25 \\
\& \& \text { user and sensors are in } \\
\text { the same Location } \\
(\mathrm{ON})\end{array}$ \\
\hline $\begin{array}{l}\text { Tweet is Hot \& \& Temp }<25 \\
\& \& \text { user and sensors are in } \\
\text { the same Location } \rightarrow(\text { OFF })\end{array}$ & $\mathrm{OFF}$ \\
\hline $\begin{array}{c}\text { Tweet is Hot \&\& Temp > } 25 \\
\& \& \text { user and sensors are in } \\
\text { the different Location -> } \\
\text { (OFF) }\end{array}$ & OFF \\
\hline $\begin{array}{c}\text { Tweet is Hot \&\& Temp <25 } \\
\& \& \text { user and sensors are in } \\
\text { the diff Location (OFF) }\end{array}$ & $\mathrm{OFF}$ \\
\hline $\begin{array}{l}\text { Tweet is cold \&\& Temp >25 } \\
\& \& \text { user and sensors are in } \\
\text { the same Location } \quad->\text { OFF }\end{array}$ & OFF \\
\hline $\begin{array}{l}\text { Tweet is Hot \&\& Temp > } 25 \\
\& \& \text { user and sensors are in } \\
\text { the diff Location } \quad->\text { ON }\end{array}$ & ON \\
\hline $\begin{array}{c}\text { Tweet is cold } \& \& \text { Temp }<25 \\
\& \& \text { user and sensors are in } \\
\text { the diff Location } \quad->\text { ON }\end{array}$ & $\mathrm{ON}$ \\
\hline $\begin{array}{c}\text { cold } \& \&>25 \& \& \text { Diff } \\
\text { Location }->\text { ON }\end{array}$ & $\mathrm{ON}$ \\
\hline $\begin{array}{c}\text { Tweet is cold \&\& Temp }<25 \\
\& \& \text { user and sensors are in } \\
\text { the diff Location }->\text { OFF }\end{array}$ & $\begin{array}{c}\text { Ice-cream } \& \&<25 \& \& \\
\text { Diff Location }->\text { ON }\end{array}$ \\
\hline
\end{tabular}


According to the experiment, we found the average of Precision is $80 \%$, Average of Recall is $80 \%$, and the average of F-Score is that reflect the sensitivity and accuracy is $80 \%$ as calculated using python scikit API as shown in Figure.17.
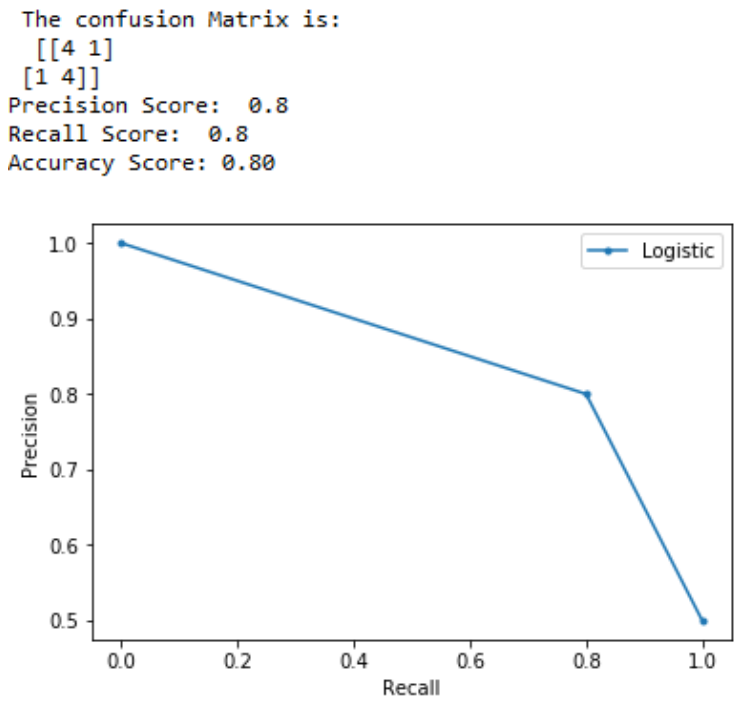

Fig. 10. Our Smart Recommender Evaluation Measurements

So the accuracy of our smart rule-based recommender system is $80 \%$ as results of the precision-recall values. On the other hand, our proposed recommender is evaluated in terms of response time that reflects the duration between the time when the user shares his status through social media platforms and the time of performing the recommended action by the actuators of the IoT applications. We performed a set of 10 experiments using different status, each one has different response time and finally, we calculated the average of response time as shown in Figure.18.

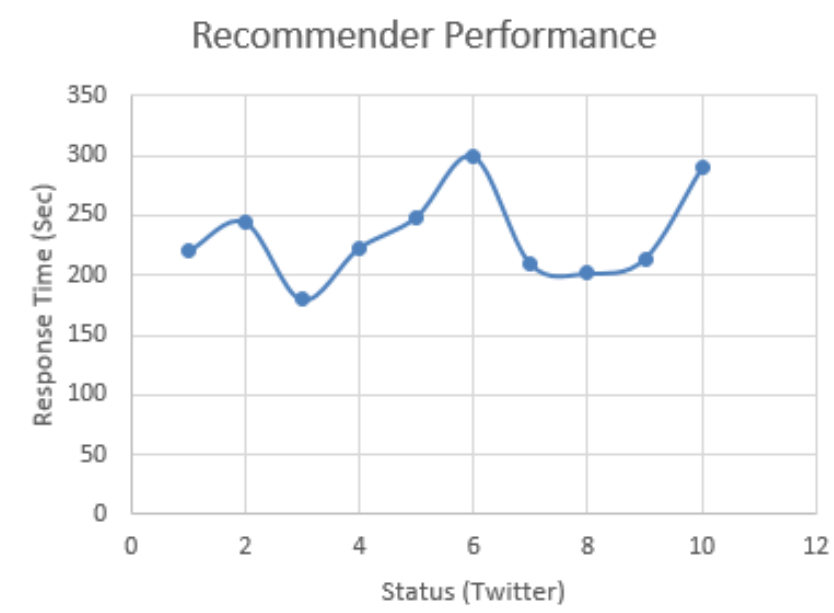

Fig. 11. Response Time (Performance rate) of our Rule-Based Social IoT Recommender System

\section{CONCLUSION AND FUTURE WORKS}

Through this research, a new social recommender-based on the semantic web, Sentiment analysis techniques, and IoT is developed. We depend on developing our algorithm on modeling the IoT resources using semantic web techniques and Ontology engineering languages such as OWL and RDF. Also, we used the sentiment analysis algorithms for analyzing user behavior on social media platforms to perform a robust reasoning tool that helped us in developing our proposed recommender. This recommendation system allows an auto recommendation for users who are actually affected by these IoT resources such as the sensors and the actuators and hence appear active on social networking platforms. Through this auto recommender, social media users can feel with the change in his surrounding environment while he updates his status through different social platforms. Hence, applying our developed recommender in real life reflects the high impact of social media in improving the IoT applications and leverage the huge amount of analyzed data of sensors and social media, the Eventually, our future works will be the focus on applying similar algorithms in other disciplines such as healthcare development and e-commerce technologies which could help users to feel better and be more flexible when they deal with different resources belong to IoT world.

\section{REFERENCES}

[1] O. Hamdan, H. Shanableh, I. Zaki, A. R. Al-Ali and a. T. Shanableh, "IoT-based interactive dual-mode smart home automation."," in IEEE International Conference on Consumer Electronics (ICCE), In 2019

[2] A. Basu, "Semantic Web, Ontology, and Linked Data," in Web Services: Concepts, Methodologies, Tools, and Applications, India, 2019, p. 22.

[3] Compton, M.; Barnaghi, P.; Bermudez, L.; GarcíACastro, R.; Corcho, O.; Cox, S.; Graybeal, J.; Hauswirth, M., "The SSN ontology of the W3C semantic sensor network incubator group.," Web Semant.Sci. Ser. Agents World Wide Web, vol. 17, p. 25-32, 2012.

[4] G. Paltoglou and M. Thelwall, "Twitter, MySpace, Digg: Unsupervised Sentiment Analysis in Social Media," ACM Transactions on Intelligent Systems and Technology (TIST), vol. 3, no. 4, 2012.

[5] S. Zander, N. Merkle, and M. Frank, "Enhancing the Utilization of IoT Devices Using Ontological Semantics and Reasoning," Procedia Computer Science, vol. 98, pp. 87-90, 2016.

[6] M. Botts and A. Robin, "Sensor Model Language (SensorML) Implementation Specification. Version 1.0. $0, " 2007$.

[7] M.Alexandra and M.Dunja, "A Framework for Semantic Enrichment of Sensor Data," Journal of Computing and Information Technology, vol. 3, p. 167-173, 2012.

[8] D. F. Barbieri, D. Braga, S. Ceri, E. Valle, and M. 
Grossniklaus, "Querying RDF Streams with CSPARQL," SIGMOD Record, vol. 39, no. 1, p. 20-26, 2010.

[9] A. M.Isen, "Positive Affect, Cognitive Processes, and Social Behavior," Advances in Experimental Social Psychology, vol. 20, pp. 203-253.

[10] L. Li, C. Zhou, J. He, J. Wang, X. Li and X. Wu, "Collective semantic behavior extraction in social networks," Journal of Computational Science- Elsevier, vol. 28, pp. 236-244, 2018.

[11] A. Khaled, S. Ouchani, and C. Chopra, "Recommendations-based on semantic analysis of social networks in learning environments," Computers in Human Behavior- Elsevier, 2018.

[12] F. G. Contratres, S. N. Alves-Souza, L. V. L. Filgueiras, and L. S. DeSouza, "Sentiment Analysis of Social Network Data for Cold-Start Relief in Recommender Systems," in World Conference on Information Systems and Technologies - WorldCIST'18 2018, Brazil.

[13] W.-C. Chien, C.-F. Lai, M. S. Hossain, and G. Muhammad, "Heterogeneous Space and Terrestrial Integrated Networks for IoT: Architecture and Challenges."," IEEE Network, vol. 33, no. 1, pp. 15-21, 2019.

[14] Sarker, K. Md, and H. Pascal, "Efficient concept induction for description logics."," in " Proceedings of the AAAI Conference on Artificial Intelligence.", 2019..

[15] S. Mehla and J. Sarika, "Rule languages for the semantic web."," in Emerging Technologies in Data Mining and Information Security., Singapore, 2019.

[16] M. H. Jooho Kim, "Social network analysis: Characteristics of online social networks after a disaster," International Journal of Information Management, 2017.

[17] V. Subramaniyaswamy, R. Logesh, M. Abejith, S Umasankar, and A. Umamakeswari, "Sentiment analysis of tweets for estimating criticality and security of events.," in Improving the Safety and Efficiency of Emergency Services: Emerging Tools and Technologies for First Responders, 2020.

[18] J. Shokeen and C. Rana, "A study on features of social recommender systems," Artificial Intelligence Review, p. 1-24, 2019.

[19] M. De, Ben, Pieter, Heyvaert, V. Ruben and D. Anastasia, "Mapping languages: analysis of comparative characteristics."," 2019.

[20] G. Brewka, Markus and Ulbricht., "Strong Explanations for Nonmonotonic Reasoning."," In Description Logic, Theory Combination, and All That, Cham, 2019.

[21] Hyun, J., Ryu, S., Lee, S.-Y. T. J. J. o. I., \& Systems, I. (2019). How to improve the accuracy of recommendation systems: Combining ratings and review texts sentiment scores. 25(1), 219-239. 